Gastroenterologe $2021 \cdot 16: 223$

https://doi.org/10.1007/s11377-021-00537-z

Angenommen: 18. Mai 2021

(c) Springer Medizin Verlag $\mathrm{GmbH}$, ein Teil von Springer Nature 2021

\section{Immuntherapie bei gastrointestinalen Tumoren}

\author{
Thomas Seufferlein ${ }^{1}$. Nisar Malek ${ }^{2}$ \\ ${ }^{1}$ Klinik für Innere Medizin I, Gastroenterologie - Endokrinologie - Nephrologie - Ernährung und \\ Stoffwechselkrankheiten, Universitätsklinikum Ulm, Ulm, Deutschland \\ ${ }^{2}$ Klinik für Innere Medizin 1, Gastroenterologie - Gastrointestinale Onkologie - Hepatologie - \\ Infektiologie und Geriatrie, Universitätsklinikum Tübingen, Tübingen, Deutschland
}

Kaum ein wissenschaftliches Gebiet in der Onkologie war so lange „High-potentialbut-low-delivery"-Thema wie die Immuntherapie. Jahrzehntelange Forschung zu tumorspezifischen Antigenen und Strategien, sich diese therapeutisch zu Nutze zu machen, zeigten bis vor Kurzem nur bescheidene klinische Erfolge. Die Idee, dass sich der Körper letztlich nur selbst besiegen kann, führte zum Erfolg und zum Nobelpreis für James P. Allison und Tasuku Honjo im Jahr 2018 für ihr Konzept einer „Hemmung der negativen Immunregulation". Tumorzellen können die Immunantwort des Körpers negativ regulieren. Dies geschieht über Immuncheckpointrezeptoren. So lässt sich z. B. durch Blockade des "cytotoxic T-lymphocyte-associated antigen $4^{\prime \prime}$ (CTLA4) auf der Oberfläche der T-Zellen die Immunreaktion des Körpers gegen Tumoren deutlich verstärken. Ein zweiter, ebenfalls negativ regulierender Checkpoint ist "programmed cell death protein 1" (PD-1). PD-1 wird ebenfalls auf Lymphozyten, aber auch auf natürlichen Killerzellen und B-Zellen exprimiert, begrenzt Entzündungsreaktionen und verhindert Autoimmunreaktionen. Tumoren nutzen PD-1-Liganden, um eine gegen den Tumor gerichtete Immunantwort zu unterbinden. Mittlerweilen sind zahlreiche Antikörper gegen PD-1 und PD-L1 bei vielen Tumorentitäten im Einsatz und bei einigen Tumoren wie dem Melanom zum klinischen Therapiestandard geworden.

Bei den gastrointestinalen Tumoren ist das Bild komplex, da wie wir zwischenzeitlich gelernt haben, dass die Effektivität der Immuncheckpointinhibitortherapie von bestimmten Faktoren abhängt wie z. B. dem Mikrosatellitenstatus des Tumors oder der Tumormutationslast. Welche Bio- marker bei welchen gastrointestinalen Tumoren eine Vorhersage des Ansprechens einer Immuntherapie bei gastrointestinalen Tumoren erlauben, ist Thema des ersten Beitrags der Gruppe um Albrecht Stenzinger und Matthias Kloor aus Heidelberg. Vakzinierung hat gerade in der COVID-Pandemie einen enormen Aufschwung erlebt und wir sehen jetzt das enorme Potenzial der neuen, RNA-basierten Vakzinierungsstrategien. Diese Strategien wurden ursprünglich für die Tumortherapie entwickelt. Auf diese und alle weiteren gängigen Vakzinierungsstrategien gehen Yacine Maringer und Juliane Walz aus Tübingen in ihrem Beitrag ein. Zwei herausragende Vertreterlnnen aus der gastrointestinalen Onkologie, Sylvie Lorenzen aus München und Alexander Stein aus Hamburg, stellen dann in ihren Beiträgen den State of the Art der Immuntherapie im oberen bzw. unteren Gastrointestinaltrakt dar und gehen auch auf aktuell laufende Studien ein. Diese Beiträge sind deshalb von großem Interesse, da die European Medicines Agency (EMA) vor wenigen Wochen Zulassungen für Immuntherapeutika bei Tumoren des gastroösophagealen Übergangs und bei mikrosatelliteninstabilen kolorektalen Karzinomen aufgrund sehr überzeugender Studiendaten erteilt hat.

\section{॥) Zahlreiche Antikörper gegen PD-1 und PD-L1 wurden bei einigen gastrointestinalen Tumoren zum klinischen Therapiestandard}

Das Thema Immuntherapie wird uns noch lange beschäftigen. Zahlreiche weitere Studienkonzepte, u.a. zu Kombinationen von Checkpointinhibitoren mit Chemotherapeutika oder „targeted agents" oder zur Verbesserung der Effektivität von Immuntherapeutika bei immunologisch „kalten" Tumoren (wie z. B. dem Pankreaskarzinom) oder in neoadjuvanten und adjuvanten Therapiesituationen sind auf dem Weg. Diese Studien werden den Stellenwert der Immuntherapie im Kontext eines multimodalen Therapiekonzepts gastrointestinaler Tumoren weiter definieren.

Ihre

\section{Nisar Malek}

Thomas Seufferlein

Interessenkonflikt. T.Seufferlein und N. Malekgeben an, dass kein Interessenkonflikt besteht.

\section{Korrespondenzadresse}

\section{Prof. Dr. Thomas Seufferlein}

Klinik für Innere Medizin I, Gastroenterologie Endokrinologie - Nephrologie - Ernährung und Stoffwechselkrankheiten, Universitätsklinikum Ulm

Albert-Einstein-Allee 23, 89081 Ulm,

Deutschland

thomas.seufferlein@uniklinik-ulm.de 